EUROPEAN JOURNAL OF PURE AND APPLIED MATHEMATICS

Vol. 14, No. 4, 2021, 1367-1378

ISSN 1307-5543 - ejpam.com

Published by New York Business Global

\title{
On Restrained Strong Resolving Domination in Graphs
}

\author{
Helyn C. Sumaoy ${ }^{1}$, Helen M. Rara ${ }^{2, *}$ \\ 1 Department of Mathematics and Statistics, College of Science and Mathematics, \\ Center of Graph Theory, Algebra, Mindanao State University-Iligan Institute of Technology, \\ 9200 Iligan City, Philippines \\ 2 Analysis-Premier Research Institute of Science and Mathematics, Mindanao State University- \\ Iligan Institute of Technology, 9200 Iligan City, Philippines
}

\begin{abstract}
A set $S \subseteq V(G)$ is a restrained strong resolving dominating set in $G$ if $S$ is a strong resolving dominating set in $G$ and $S=V(G)$ or $\langle V(G) \backslash S\rangle$ has no isolated vertex. The restrained strong resolving domination number of $G$, denoted by $\gamma_{r s R}(G)$, is the smallest cardinality of a restrained strong resolving dominating set in $G$. In this paper, we present characterizations of the restrained strong resolving dominating sets in the join, corona and lexicographic product of two graphs and determine the exact value of the restrained strong resolving domination number of each of these graphs.
\end{abstract}

2020 Mathematics Subject Classifications: 05C69

Key Words and Phrases: restrained strong resolving dominating set, restrained strong resolving domination number, join, corona, lexicographic product

\section{Introduction}

Domination in graphs was first introduced by C. Berge in 1958 [1]. There are many studies involving domination and its variations. Slater [8] introduced and studied the concept of resolving set. In 2003, Robert Brigham et al. [18] linked the concepts of resolving and domination. In their article, they defined a resolving dominating set as a set that is both resolving and dominating. Resolving sets and resolving dominating sets were further studied in $[2,3]$. Oellermann, O. R, and Peters-Fransen, J. [6] introduced and studied the concept of strong resolving set.

Domke et. al [7] introduced and investigated the concept of restrained domination in graphs.

Khuller, et. al. [11] introduced the concept of metric dimension and this has grown to become an interesting topic in graph theory. In line with this, Sebo and Tannier [13] introduced the concept of strong metric dimension, a concept which is more restrictive than

*Corresponding author.

DOI: https://doi.org/10.29020/nybg.ejpam.v14i4.4112

Email addresses: helyn.sumaoy@g.msuitt.edu.ph (H. Sumaoy),

helen.rara@g.msuitt.edu.ph, helenrara@gmail.com (H. Rara) 
metric dimension. After its introduction, Slater in [8] studied it and discovered its usefulness when working with the United States sonar and Coast Guard Loran (long range aids to navigation) stations. Its applications arise in many diverse fields including chemistry, for representing chemical compounds [10], the robot navigation [11] and geographical routing protocols [12], to name a few. In [13], an invariant called the strong metric dimension, was presented where the authors illustrated its application to combinatorial search. Along with the increasing discovery of its applications, theoretical studies on this invariant also appear in several number of other papers including [14], [15], [16], [17]. This paper intends to generate additional theoretical results and help widen the pool of existing studies from where new researchers may draw new insights and directions for further investigation.

In this study, we investigate the concept of restrained strong resolving domination in the join, corona, and lexicographic product of graphs. Readers are referred to [9] for elementary Graph Theory concepts.

Let $G=(V(G), E(G))$ be a graph. The open neighborhood of $v \in V(G)$ is $N_{G}(v)=\{u \in V(G): u v \in E(G)\}$. An element $u \in N_{G}(v)$ is called a neighbor of $v$. The closed neighborhood $v \in V(G)$ is $N_{G}[v]=N_{G}(v) \cup\{v\}$. Thus, the degree of $v \in V(G)$ is given by $\operatorname{deg}_{G}(v)=\left|N_{G}(v)\right|$. For $S \subseteq V(G), N_{G}(S)=\bigcup_{v \in S} N_{G}(v)$ and $N_{G}[S]=\bigcup_{v \in S} N_{G}[v]$.

A clique in a graph $G$ is a complete induced subgraph. A set $C \subseteq V(G)$ is called a superclique in $G$ if $\langle C\rangle$ is a clique and for every pair of distinct vertices $u, v \in C$, there exists $w \in V(G) \backslash C$ such that $w \in N_{G}(u) \backslash N_{G}(v)$ or $w \in N_{G}(v) \backslash N_{G}(u)$. A superclique $C$ is maximum in $G$ if $|C| \geq\left|C^{*}\right|$ for all supercliques $C^{*}$ in $G$. The superclique number $\omega_{S}(G)$ of $G$ is the cardinality of a maximum superclique in $G$. A superclique $C$ is called a dominated superclique if for every $u \in C$ there exists $v \in V(G) \backslash C$ such that $u v \in E(G)$. The dominated superclique number $\omega_{D S}(G)$, of $G$ is the cardinality of a maximum dominated superclique in $G$.

A vertex $x$ of a graph $G$ is said to resolve two vertices $u$ and $v$ of $G$ if $d_{G}(x, u) \neq d_{G}(x, v)$. For an ordered set $W=\left\{x_{1}, \ldots, x_{k}\right\} \subseteq V(G)$ and a vertex $v$ in $G$, the $k$-vector

$$
r_{G}(v / W)=\left(d_{G}\left(v, x_{1}\right), d_{G}\left(v, x_{2}\right), \ldots, d_{G}\left(v, x_{k}\right)\right)
$$

is called the representation of $v$ with respect to $W$. The set $W$ is a resolving set for $G$ if and only if no two vertices of $G$ have the same representation with respect to $W$. The metric dimension of $G$, denoted by $\operatorname{dim}(G)$, is the minimum cardinality over all resolving sets of $G$. A resolving set of cardinality $\operatorname{dim}(G)$ is called a basis.

A set $S \subseteq V(G)$ of vertices of $G$ is a dominating set if every $u \in V(G) \backslash S$ is adjacent to at least one vertex $v \in S$. The domination number of a graph $G$, denoted by $\gamma(G)$, is given by $\gamma(G)=\min \{|S|: S$ is a dominating set of $G\}$.

A subset $S \subseteq V(G)$ is a strong resolving dominating set of $G$ if $S$ is a dominating set and for every pair of vertices $u, v \in V(G)$, there exists a vertex $w \in S$ such that $u \in I_{G}[v, w]$ or $v \in I_{G}[u, w]$. The smallest cardinality of a strong resolving dominating set of $G$ is called the strong resolving domination number of $G$ and is denoted by $\gamma_{s R}(G)$. A strong resolving dominating set of cardinality $\gamma_{s R}(G)$ is called a $\gamma_{s R^{-}}$set of $G$. 
A set $S \subseteq V(G)$ is a restrained dominating set of $G$ if $S$ is a dominating set of $G$ and for every $v \in V(G) \backslash S$ there exists $u \in(V(G) \backslash S) \cap N_{G}(v)$. Equivalently, a dominating subset $S$ of $V(G)$ is a restrained dominating set of graph $G$ if $S=V(G)$ or $\langle V(G) \backslash S\rangle$ has no isolated vertex. The restrained domination number of $G$, denoted by $\gamma_{r}(G)$ is the minimum cardinality of a restrained dominating set of $G$. Any restrained dominating set of $G$ of cardinality $\gamma_{r}(G)$ is referred to as a $\gamma_{r}$-set of $G$.

The join of two graphs $G$ and $H$ is the graph $G+H$ with vertex set $V(G+H)=V(G) \dot{\cup} V(H)$ and edge set $E(G+H)=E(G) \dot{\cup} E(H) \cup\{u v: u \in$ $V(G), v \in V(H)\}$. The corona of two graphs $G$ and $H$, denoted by $G \circ H$, is the graph obtained by taking one copy of $G$ of order $n$ and $n$ copies of $H$, and then joining every vertex of the $i$ th copy of $H$ to the $i$ th vertex of $G$. For $v \in V(G)$, denote by $H^{v}$ the copy of $H$ whose vertices are attached one by one to the vertex $v$. Subsequently, denote by $v+H^{v}$ the subgraph of the corona $G \circ H$ corresponding to the join $\langle\{v\}\rangle+H^{v}, v \in V(G)$. The lexicographic product of two graphs $G$ and $H$, denoted by $G[H]$, is the graph with vertexset $V(G[H])=V(G) \times V(H)$ such that $\left(u_{1}, u_{2}\right)\left(v_{1}, v_{2}\right) \in E(G[H])$ if either $u_{1} v_{1} \in E(G)$ or $u_{1}=v_{1}$ and $u_{2} v_{2} \in E(H)$.

\section{Preliminary Results}

This section presents some of the important known results in strong resolving domination of graphs and some properties of restrained strong resolving dominating set in a graph.

Theorem 1. [3] Let $G$ be a nontrivial connected graph of order $n$ with $\gamma(G) \neq 1$ and $K_{1}=\langle v\rangle$. Then $S \subseteq V\left(K_{1}+G\right)$ is a strong resolving dominating set of $K_{1}+G$ if and only if $S=V(G)$; or $S=V\left(K_{1}+G\right) \backslash C$ or $S=V(G) \backslash C^{*}$ where $C$ is a superclique and $C^{*}$ is dominated superclique in $G$.

Theorem 2. [3] Let $G$ be a nontrivial connected graph of order $n$ with $\gamma(G)=1$ and $K_{1}=\langle v\rangle$. Then $S \subseteq V\left(K_{1}+G\right)$ is a strong connected resolving dominating set of $K_{1}+G$ if and only if $S=V(G)$ or $S=V\left(K_{1}+G\right) \backslash C$ or

$$
S=\left(V(G) \backslash C^{*}\right) \cup\left\{x \in C^{*}: \operatorname{deg}(x)=n-1\right\}
$$

where $C$ and $C^{*}$ are superclique and dominated superclique, respectively in $G$.

Theorem 3. [3] Let $K_{1}=\langle v\rangle$ and $G$ be a disconnected graph whose components are $G_{i}$ for $i=1,2, \ldots, m$. A proper subset $S$ of $V\left(K_{1}+G\right)$ is a strong resolving dominating set of $K_{1}+G$ if and only if $S=V(G)$ or $S=V(G) \backslash C_{i}^{*}$ or $S=V\left(K_{1}+G\right) \backslash C_{i}$ where $C_{i}$ is a superclique in $G_{i}$, for $i=1,2, \ldots, m$ and $C_{i}^{*}$ is a dominated superclique of $G_{i}$.

Theorem 4. [3] Let $G$ be a nontrivial connected graph and $H$ a connected graph. A proper subset $S$ of $V(G \circ H)$ is a strong resolving dominating set of $G \circ H$ if and only if one of the following holds: 
(i) $S=A \cup\left(\bigcup_{u \in V(G)} V\left(H^{u}\right)\right)$ where $A \subseteq V(G)$;

(ii) $S=A \cup\left(\bigcup_{u \in V(G) \backslash\{v\}} V\left(H^{u}\right)\right) \cup B_{v}$ for a unique vertex $v$ in $G$, where

$A \subseteq V(G) \backslash\{v\}$ and $B_{v}$ is a strong resolving dominating set of $H^{v}$ if $\gamma(H)=1$ or $B^{v}$ is a strong resolving dominating set of $\langle v\rangle+H^{v}$ if $\gamma(H) \neq 1$.

Theorem 5. [3] Let $G$ and $H$ be non-trivial connected graphs of orders $m$ and $n$, respectively. A proper subset $S$ of $V(G+H)$ is a strong resolving dominating set of $G+H$ if and only if at least one of the following is satisfied:

(i) $S=V(G+H) \backslash C_{G}$ where $C_{G}$ is a superclique of $G$.

(ii) $S=V(G+H) \backslash C_{H}$ where $C_{H}$ is a superclique of $G$.

(iii) If $\gamma(G)=1$ and $\gamma(H)=1$,

$S=\left[V(G+H) \backslash\left(C_{G} \cup C_{H}\right)\right] \cup\left\{z \in C_{G}: \operatorname{deg}_{G}(z)=m-1\right\}$ or

$S=\left[V(G+H) \backslash\left(C_{G} \cup C_{H}\right)\right] \cup\left\{w \in C_{H}: \operatorname{deg}_{H}(w)=n-1\right\}$

where $C_{G}$ and $C_{H}$ are supercliques in $G$ and $H$, respectively.

(iv) If $\gamma(G) \neq 1$ and $\gamma(H) \neq 1$,

$S=\left[V(G+H) \backslash\left(C_{G} \cup C_{H}\right)\right]=\left(V(G) \backslash C_{G}\right) \cup\left(V(H) \backslash C_{H}\right)$

where $C_{G}$ and $C_{H}$ are supercliques in $G$ and $H$, respectively.

Lemma 1. [2] Let $G$ be a nontrivial connected graph with $\operatorname{diam}(G) \leq 2$. Then $S=V(G) \backslash C$ is a strong resolving set of $G$ if and only if $C=\varnothing \quad$ or $\quad C \quad$ is a superclique in $G$. In particular, $\operatorname{sdim}(G)=|V(G)|-\omega_{S}(G)$.

Theorem 6. [2] Let $G=K_{n}$ for $n>1$ and $\mathrm{H}$ a nontrivial connected graph with $\gamma(H) \neq 1$. A subset $S$ of $V(G[H])$ is a strong resolving set of $G[H]$ if and only $S=V(G[H]) \backslash(A \times C)$, where $A$ is a subset of $V(G)$ and $C=\varnothing$ or $C$ is a superclique in $H$.

Remark 1. Every restrained strong resolving dominating set of a connected graph $G$ is a strong resolving dominating set. Hence, $\gamma_{s R}(G) \leq \gamma_{r s R}(G)$. Also, every restrained strong resolving dominating set of $G$ is a restrained dominating set. Thus, $\gamma_{r}(G) \leq \gamma_{r s R}(G)$.

Remark 2. For any connected graph $G$ of order $n, 1 \leq \gamma_{r s R}(G) \leq n$. Moreover, $\gamma(G)=1$ if and only if $G$ is a non-trivial graph and $\gamma_{r s R}\left(K_{n}\right)=n$ for $n \geq 1$.

Proposition 1. Let $G$ be a nontrivial connected graph with $\operatorname{diam}(G) \leq 2$. Then $S \subseteq V(G)$ is a restrained strong resolving dominating set of $G$ if and only if $S=V(G) \backslash C$ where $C=\varnothing$ or $C$ is a nonsingleton dominated superclique in $G$. In particular,

$$
\gamma_{r s R}(G)=|V(G)|-\omega_{D S}(G)
$$


Proof: Let $S$ be a restrained strong resolving dominating set of $G$. Let $C=V(G) \backslash S$. Then, $S=V(G) \backslash C$. Since $S$ is a strong resolving, by Lemma $1, C=\varnothing$ or $C$ is a superclique in $G$. Since $S$ is restrained dominating, $S=V(G)$ or $V(G) \backslash S$ has no isolated vertex. Thus, $C=\varnothing$ or $C$ is a singleton dominated superclique in $G$.

The converse follows immediately from Lemma 1, definition of dominated superclique and definition of restrained dominating set.

Suppose $S$ is a $\gamma_{r s}$-set of $G$. Then, $S=V(G) \backslash C$ where $C$ is a nonsingleton dominated superclique in $G$ and $|C|=\omega_{D S}(G)$. Thus,

$$
\gamma_{r s R}(G)=|S|=|V(G)|-|C|=|V(G)|-\omega_{D S}(G) .
$$

\section{Restrained Strong Resolving Domination in the Join of Graphs}

Theorem 7. Let $G$ be a nontrivial connected graph of order $n$ with $\gamma(G) \neq 1$. Then $S \subseteq V\left(K_{1}+G\right)$ is a restrained strong resolving dominating set of $K_{1}+G$ if and only if $S=V(G) \backslash C$ or $S=V\left(K_{1}+G\right) \backslash C^{*}$ where $C$ is a non-singleton dominated superclique and $C^{*}=\varnothing$ or $C^{*}$ is non-singleton dominated superclique of $G$.

Proof: Let $S$ be a restrained strong resolving set of $K_{1}+G$. Since $S$ is strong resolving dominating set by Theorem $1, S=V(G)$ or $S=V(G) \backslash C$ or $S=V\left(K_{1}+G\right) \backslash C^{*}$ where $C$ is a dominated superclique and $C^{*}$ is a superclique in $G$. Since $S$ is restrained dominating, $S=V\left(G+K_{1}\right)$ or $V\left(G+K_{1}\right) \backslash S$ has no isolated vertex. Hence, $S \neq V(G)$ and $S=V(G) \backslash C$ or $S=V\left(K_{1}+G\right) \backslash C^{*}$ where $C$ is dominated supeclique and $C^{*}=\varnothing$ or $C^{*}$ is a nonsingleton superclique of $G$.

The converse follows immediately from Theorem 1, definitions of dominated superclique and restrained dominating set of a graph.

Theorem 8. Let $G$ be a nontrivial connected graph of order $n$ with $\gamma(G)=1$ and $K_{1}=\langle v\rangle$. Then $S \subseteq V\left(K_{1}+G\right)$ is a restrained strong resolving dominating set of $K_{1}+G$ if and only if $S=V\left(K_{1}+G\right) \backslash C$ or $S=\left(V(G) \backslash C^{*}\right) \cup\left\{x \in C^{*}: \operatorname{deg}_{G}(x)=n-1\right\}$ where $C=\varnothing$ or $C$ is nonsingleton superclique of $G$ and $C^{*}$ is a superclique of $G$.

Proof: Let $S$ be a restrained strong resolving dominating set of $K_{1}+G$. Then by Theorem $2, S=V(G)$ or $S=V\left(K_{1}+G\right) \backslash C$ or $S=\left(V(G) \backslash C^{*}\right) \cup\left\{x \in C^{*}: \operatorname{deg}_{G}(x)=\right.$ $n-1$ \} where $C$ and $C^{*}$ are superclique and dominated superclique of $G$, respectively. Since $S$ is restrained dominating, $S=V\left(K_{1}+G\right)$ or $V\left(K_{1}+G\right) \backslash S$ has no isolated vertex. Thus, $S \neq V(G), C=\varnothing$ or $C$ is non-singleton dominated superclique of $G$ and $C^{*}$ is a superclique of $G$.

The converse follows immediately from Theorem 2, definitions of superclique and restrained dominating set of a graph.

The next results follow immediately from Theorem 7 .

Corollary 1. Let $G$ be a nontrivial connected graph of order $n$. Then

$$
\gamma_{r s R}\left(K_{1}+G\right)= \begin{cases}n-\omega_{S}(G)+1, & \text { if } \gamma(G)=1 \\ n-\omega_{D S}(G), & \text { if } \gamma(G) \neq 1\end{cases}
$$


Corollary 2. Let $G$ be a connected graph with $\operatorname{diam}(G) \leq 2$. Then

$$
\gamma_{r s R}\left(K_{1}+G\right)= \begin{cases}\operatorname{sdim}(G)+1, & \text { if } \gamma(G)=1 \\ \gamma_{s R}(G) & \text { if } \gamma(G) \neq 1\end{cases}
$$

Corollary 3. Let $P_{n}=\left[v_{1}, v_{2}, \ldots, v_{n}\right]$ and $C_{m}=\left[c_{1}, c_{2}, \ldots c_{m}, c_{1}\right]$ where $n, m \geq 3$.

(i) The sets $V\left(P_{n}\right) \backslash\left\{v_{i}, v_{i+1}\right\}$ for $i=2,3, \ldots, n-2, V\left(P_{n}\right) \backslash\left\{v_{j}\right\}$ for $j=1,2, \ldots, n$ and $V\left(P_{n}\right) \cup\{v\} \backslash\left\{v_{k}, v_{k+1}\right\}$ for $k=1,2, \ldots, n-1$ are the restrained strong resolving dominating sets of $\langle v\rangle+P_{n}$.

(ii) The sets $V\left(C_{m}\right) \backslash\left\{c_{i}, c_{i+1}\right\},\left(V\left(C_{m}\right) \cup\{v\}\right) \backslash\left\{c_{i}, c_{i+1}\right\}, V\left(C_{m}\right) \backslash\left\{v_{i}\right\}, V\left(C_{m}\right) \backslash\left\{v_{m}\right\}$, $V\left(C_{m}\right) \backslash\left\{c_{i}, c_{m}\right\}$ and $\left(V\left(C_{m}\right) \cup\{v\} \backslash\left\{c_{1}, c_{m}\right\}\right)$ for $i=1,2, \ldots, m-1$, are the restrained strong resolving dominating sets of $\langle v\rangle+C_{n}$.

Theorem 9. Let $G$ be a disconnected graph whose components are $G_{i}$ for $i=1,2, \ldots, n$. A subset $S$ of $V\left(K_{1}+G\right)$ is a restrained strong resolving dominating set of $K_{1}+G$ if and only if $S=V(G) \backslash C_{i} \quad$ or $S=V\left(K_{1}+G\right) \backslash C_{i}^{*}$ where $C_{i}$ is a dominated superclique of $G_{i}$ and $C_{i}^{*}=\varnothing$ or $C_{i}^{*}$ is a nonsingleton superclique of $G$.

Proof: Let $S$ be a restrained strong resolving dominating set of $K_{1}+G$. Then by Theorem $3 \quad S=V(G) \quad$ or $\quad S=V(G) \backslash C_{i} \quad$ or $S=V\left(K_{1}+G\right) \backslash C_{i}^{*}$ where $C_{i}$ is a dominated superclique of $G_{i}$ and $C_{i}^{*}=\varnothing$ or $C_{i}^{*}$ is a nonsingleton superclique in $G_{i}$. Since $S$ is restrained dominating, $S=V\left(K_{1}+G\right)$ or $V\left(K_{1}+G\right) \backslash S$ has no isolated vertex. Hence, $S \neq V(G)$ and $C_{i}^{*}=\varnothing$ or $C_{i}^{*}$ is nonsingleton. Therefore, $S=V(G) \backslash C_{1}$ or $S=V\left(K_{1}+G\right) \backslash C_{i}^{*}$ where $C_{i}$ is a dominated superclique in $G_{i}$ and $C_{i}^{*}$ is a nonsingleton superclique in $G_{i}$.

The converse follows immediately from Theorem 3, definitions of dominated superclique and restrained dominating set of a graph.

Corollary 4. Let $G_{i}$ be connected graphs of order $n_{i}$ and $G$ be a disconnected graph whose components are $G_{i}$ for $i=1,2, \ldots, m$. Then, $\gamma_{r s R}\left(K_{1}+G\right)=\sum_{i=1}^{m} n_{i}-r_{G}$ where $r_{G}=\max \left\{\max \left\{\omega_{D S}\left(G_{i}\right)\right\}, \max \left\{\omega_{S}\left(G_{i}\right)+1\right\}: i=1,2, \ldots, m\right\}$.

In the join of two graphs $G$ and $H$, the results Theorem 7 and Theorem 8 have already considered the case when $G$ or $H$ is trivial. Hence, the next results, considered the characterizations of the restrained strong resolving dominating sets of nontrivial connected graphs $G$ and $H$.

Theorem 10. Let $G$ and $H$ be nontrivial connected graphs of orders $m$ and $n$, respectively. A subset $S$ of $V(G+H)$ is a restrained strong resolving dominating set of $G+H$ if and only if at least one of the following is satisfied:

(i) $S=V(G+H) \backslash C_{G}$ where $C_{G}$ is a nonsingleton superclique of $G$.

(ii) $S=V(G+H) \backslash C_{H}$ where $C_{H}$ is a nonsingleton superclique of $G$. 
(iii) If $\gamma(G)=1$ and $\gamma(H)=1$,

$S=\left[V(G+H) \backslash\left(C_{G} \cup C_{H}\right)\right] \cup\left\{z \in C_{G}: \operatorname{deg}_{G}(z)=m-1\right\} \quad$ or

$S=\left[V(G+H) \backslash\left(C_{G} \cup C_{H}\right)\right] \cup\left\{w \in C_{H}: \operatorname{deg}_{H}(w)=n-1\right\}$

where $C_{G}$ and $C_{H}$ are supercliques in $G$ and $H$, respectively.

(iv) If $\gamma(G) \neq 1$ and $\gamma(H) \neq 1$,

$S=\left[V(G+H) \backslash\left(C_{G} \cup C_{H}\right)\right]=\left(V(G) \backslash C_{G}\right) \cup\left(V(H) \backslash C_{H}\right)$

where $C_{G}$ and $C_{H}$ are supercliques in $G$ and $H$, respectively.

Proof: Let $S$ be a restrained strong resolving dominating set of $G+H$. By Theorem 5

(a) $S=V(G+H) \backslash C_{G}$ where $C_{G}$ is a superclique of $G$.

(b) $S=V(G+H) \backslash C_{H}$ where $C_{H}$ is a superclique of $G$.

(c) If $\gamma(G)=1$ and $\gamma(H)=1$,

$S=\left[V(G+H) \backslash\left(C_{G} \cup C_{H}\right)\right] \cup\left\{z \in C_{G}: \operatorname{deg}_{G}(z)=m-1\right\} \quad$ or

$S=\left[V(G+H) \backslash\left(C_{G} \cup C_{H}\right)\right] \cup\left\{w \in C_{H}: \operatorname{deg}_{H}(w)=n-1\right\}$

where $\left\langle C_{G}\right\rangle$ and $C_{H}$ are supercliques in $G$ and $H$, respectively.

(d) If $\gamma(G) \neq 1$ and $\gamma(H) \neq 1$,

$S=\left[V(G+H) \backslash\left(C_{G} \cup C_{H}\right)\right]=\left(V(G) \backslash C_{G}\right) \cup\left(V(H) \backslash C_{H}\right)$

where $C_{G}$ and $C_{H}$ are supercliques in $G$ and $H$, respectively.

Since $S$ is a restrained dominating set of $G+H$, (i), (ii), (iii), (iv) hold.

The converse immediately follows from Theorem 5 and from definition of restrained dominating set of a graph.

Corollary 5. Let $G$ and $H$ be nontrivial connected graphs of orders $m$ and $n$, respectively. Then

$$
\operatorname{sdim}(G+H)=\left\{\begin{array}{lc}
\left(m-\omega_{s}(G)\right)+\left(n-\omega_{s}(H)\right)+1, & \text { if } \gamma(G)=1 \\
& \text { or } \gamma(H)=1 \\
\left(m-\omega_{s}(G)\right)+\left(n-\omega_{s}(H)\right), & \text { if } \gamma(G) \neq 1 \\
& \text { and } \gamma(H) \neq 1
\end{array}\right.
$$

\section{Restrained Strong Resolving Domination in the Corona of Graphs}

This section gives characterization of the restrained strong resolving dominating sets in the corona of graphs as well as its restrained strong resolving domination number.

Theorem 11. Let $G$ be a nontrivial connected graph and $H$ a connected graph. A proper subset $S \subseteq V(G \circ H)$ of a restrained strong resolving dominating set of $G \circ H$ if and only if one of the following holds:

(i) $S=A \cup\left(\bigcup_{u \in V(G)} V\left(H^{u}\right)\right)$ where $A \subseteq V(G)$ and $V(G) \backslash A$ has no isolated vertex. 
(ii) $S=A \cup\left(\bigcup_{u \in V(G) \backslash\{v\}} V\left(H^{u}\right)\right) \cup B_{v}$ for a unique vertex $v$ in $G$, where $A=V(G) \backslash\{v\}$ or $V(G) \backslash(A \cup\{v\})$ has no isolated vertex and $B_{v}$ is a strong resolving dominating set of $H^{v}+\langle v\rangle$ if $v \in S$ and $B_{v}$ is a strong resolving set where $V\left(H^{v}\right) \backslash B_{v}$ has no isolated vertex if $v \in S$.

Proof: Suppose $S$ is a restrained resolving dominating set of $G \circ H$. Then $S$ is a strong resolving dominating and by Theorem 4 one of the following holds:

(a) $S=A \cup\left(\bigcup_{u \in V(G)} V\left(H^{u}\right)\right)$ where $A \subseteq V(G)$;

(b) $S=A \cup\left(\bigcup_{u \in V(G) \backslash\{v\}} V\left(H^{u}\right)\right) \cup B_{v}$ for a unique vertex $v$ in $G$, where $A \subseteq V(G) \backslash\{v\}$ and $B_{v}$ is a strong resolving dominating set of $H^{v}$ if $\gamma(H)=1$ or $B_{v}$ is a strong resolving dominating set of $\langle v\rangle+H^{v}$ if $\gamma(H) \neq 1$.

Suppose (a) holds. Since $S$ is a proper restrained dominating subset of $G \circ H, V(G \circ$ $H) \backslash S=V(G) \backslash A$ has no isolated vertex. Thus, (i) holds. On the other hand if (b) holds, then since $S$ is a restrained dominating set and $V(G \circ H) \backslash S=$ $(V(G) \backslash A) \cup\left(V\left(H^{v}+\langle v\rangle\right) \backslash B_{v}\right), A=V(G) \backslash\{v\}$ or $V(G) \backslash(A \cup\{v\})$ has no isolated vertex and $B_{v}$ is a strong resolving dominating set of $H^{v}+\langle v\rangle$. Since $v \in S, V\left(H^{v}\right) \backslash B_{v}$ has no isolated vertex and $B_{v}$ is a strong resolving dominating set of $H^{v}+\langle v\rangle$ if $v \in S$ and $B_{v}$ is strong resolving set of $H^{v}$ and $V\left(H^{v}\right) \backslash B_{v}$ has no isolated vertex if $v \in S$. Hence, (ii) holds.

Conversely, suppose (i) and (ii) hold. By Theorem 4, $S$ is a strong resolving dominating set of $G \circ H$. If (i) holds, then $V(G \circ H) \backslash S=V(G) \backslash A$ has no isolated vertex. If (ii) holds then $V(G \circ H) \backslash S=(V(G) \backslash A) \cup\left(V\left(H^{v}+\langle v\rangle\right) \backslash B_{v}\right)$. Since $A=V(G) \backslash\{v\}$ or $V(G) \backslash(A \cup\{v\})$ has no isolated vertex, $V(G+H) \backslash S$ has no isolated vertex. In either case, $V(G \circ H) \backslash S$ has no isolated vertex. Therefore $S$ is a restrained strong resolving dominating set of $G \circ H$.

Corollary 6. Let $G$ and $H$ be nontrivial connected graphs of orders $m$ and $n$, respectively. Then, $\gamma_{r s R}(G \circ H)=(m-1) n+\gamma_{s R}\left(H+K_{1}\right)$.

Proof: Let $S$ be a $\gamma_{r s R}$-set of $G \circ H$. Then by Theorem 11 (ii),

$$
S=\bigcup_{u \in V(G) \backslash\{v\}} V\left(H^{u}\right) \bigcup B_{v}
$$

for a unique vertex $v$ in $G$ and $B_{v}$ is a strong resolving dominating set of $H^{v}$. Hence,

$$
\begin{aligned}
\gamma_{r s R}(G \circ H)=|S| & =|V(H)||V(G) \backslash\{v\}|+\left|B_{v}\right| \\
& \geq(m-1) n+\gamma_{s R}(H)
\end{aligned}
$$


Let $C_{v}$ be a minimum strong resolving dominating set of $H^{v}$. For a unique vertex $v \in V(G)$, let $\left\langle B_{v}\right\rangle \cong\left\langle C_{v}\right\rangle$. Then by Theorem $11 S=\left(\bigcup_{u \in V(G) \backslash\{v\}} V\left(H^{u}\right)\right) \cup B_{v}$ is a restrained strong resolving dominating set of $G \circ H$. Thus,

$$
\begin{aligned}
\gamma_{r s R}(G \circ H) & \leq|S| \\
& =\left|\bigcup_{u \in V(G) \backslash\{v\}} V\left(H^{u}\right)\right|+\left|B_{v}\right| \\
& =(m-1)(n)+\left|C_{v}\right| \\
& =(m-1)(n)+\gamma_{s R}(H)
\end{aligned}
$$

Therefore, $\gamma_{r s R}(G \circ H)=(m-1) n+\gamma_{s R}(H)$.

\section{Restrained Strong Resolving Domination in the Lexicographic Product of Graphs}

Lemma 2. Let $G=K_{n}$ for $n>1$ and $H$ a nontrivial connected graph with $\gamma(H) \neq 1$. Then $A \times C \subseteq V(G[H])$ is a dominated superclique in $G[H]$ if and only if $A$ is a nonempty subset of $V(G)$ and $C$ is a superclique in $H$.

Proof: Let $G=K_{n}$ for $n>1$ and $H$ a nontrivial connected graph with $\gamma(H) \neq 1$. Suppose $A \times C \subseteq V(G[H])$ is a dominated superclique in $G[H]$. By a Lemma in [3], $A$ is a nonempty subset of $V(G)$ and $C$ is a superclique in $H$.

Conversely, suppose $A \subseteq V(G), A \neq \varnothing$ and $C$ is a superclique in $H$. Then $A \times C \subseteq V(G[H])$ is a superclique in $G[H]$ by a Lemma in [3]. We show that $A \times C$ is a dominated superclique. Let $(a, b) \in A \times C$. Then $a \in A$ and $b \in C$. Since $\gamma(H) \neq 1$, there exists $d \in V(H) \backslash N_{H}(b)$. Hence, $d \notin C$. Since $G=K_{n}$ for $n>1$, a vertex $v \in V\left(K_{n}\right) \backslash\{a\}$ exists where $a v \in E\left(K_{n}\right)$. Thus, $(v, d) \notin A \times C$ and $(a, b)(v, d) \in E(G[H])$. Therefore, $A \times C$ is a dominated superclique in $G[H]$.

Theorem 12. Let $G=K_{n}$ for $n>1$ and $H$ a nontrivial connected graph with $\gamma(H) \neq 1$. A subset $S$ of $V(G[H])$ is a restrained strong resolving dominating set of $G[H]$ if and only if $S=V(G[H]) \backslash(A \times C)$ and one of the following is satisfied:

(i) $A \subseteq V(G)$ and $C=\varnothing$

(ii) $A$ is singleton subset of $V(G)$ and $C$ is a nonsingleton suerclique in $H$.

(iii) $A$ is nonempty nonsingleton subset of $V(G)$ and $C$ is a superclique in $H$.

Proof: Let $S$ be a restrained strong resolving dominating set of $G[H]$. By Theorem 6 , $S=V(G[H]) \backslash(A \times C)$ where $A$ is a subset of $V(G)$ and $C=\varnothing$ or $C$ is a superclique in $H$. Since $S$ is restrained strong resolving dominating, $S=V(G[H])$ or $V(G[H]) \backslash S$ has 
no isolated vertex. If $S=V(G[H])$ then $A \times C=\varnothing$, showing that $A \subseteq V(G)$ and $C=\varnothing$. Thus, (i) holds. If $V(G[H]) \backslash S$ has no isolated vertex, then $A \times C$ is a nonsingleton dominated superclique in $G[H]$. This implies that $A$ is a singleton subset of $V(G)$ and $C$ is a nonsingleton superclique in $H$ or $A$ is nonempty nonsingleton subset of $V(G)$ and $C$ is a superclique in $H$. Hence (ii) or (iii) holds.

For the converse, suppose $S=V(G[H]) \backslash(A \times C)$, where $A$ and $C$ satisfy (i),(ii) or (iii). Then, either $A \times C=\varnothing$ or by Lemma $2, A \times C$ is a nonsingleton dominated superclique in $G[H]$. By Theorem $6, S$ is a strong resolving set in $G[H]$. Since $A \times C$ is a dominated superclique, $S$ is a strong resolving dominating set of $G[H]$. If (i) is true, then $A \subseteq V(G)$ and $C=\varnothing$, that is, $A \times C=\varnothing$ and $S=V(G[H])$. If (ii) or (iii) is satisfied, then $V(G[H]) \backslash S$ has no isolated vertex. Therefore, $S$ is a restrained strong resolving dominating set $G[H]$.

Lemma 3. Let $G=K_{n}$ for $n>1$ and $H$ a nontrivial connected graph with $\gamma(H)=1$. Then $A \times C \subseteq V(G[H])$ is a dominated superclique of $G[H]$ if and only if $A$ is a nonempty subset of $V(G)$ and $C$ is a superclique in $H$ such that $|A|=1$ whenever $C \cap C^{*} \neq \varnothing$ for some $\gamma$-set $C^{*}$ of $H$.

Proof: Suppose $A \times C \subseteq V(G[H])$ is a dominated superclique in $G[H]$. Then $A \times C \neq \varnothing$. By a Lemma in [3], $A$ is a nonempty subset of $V(G)$ and $C$ is a superclique in $H$ such that $|A|=1$ whenever $C \cap C^{*} \neq \varnothing$ for some $\gamma$-set $C^{*}$ of $H$.

Conversely, suppose $A \subseteq V(G)$ and $A \neq \varnothing$ and $C$ is a superclique in $H$ such that $|A|=1$ whenever $C \cap C^{*} \neq \varnothing$ for some $\gamma$-set $C^{*}$ of $H$. Then by a Lemma in [3], $A \times C$ is a superclique in $G[H]$. We show that $A \times C$ is a dominated superclique in $G[H]$. Let $(a, b) \in A \times C$. Then $a \in A$ and $b \in C$. If $C \cap C^{*}=\varnothing$ for all $\gamma$-set $C^{*}$ of $H$, then there exists $d \in V(H) \backslash N_{H}(b)$. Thus, $d \notin C$. Since $G=K_{n}$ for $n>1$, a vertex $y \in\left(V\left(K_{n}\right) \backslash\{a\}\right) \cup N_{G}(a)$ exists. This implies that

$$
(y, d) \in[V(G[H]) \backslash(A \times C)] \cap N_{G[H]}((a, b)) .
$$

Suppose, that $C \cap C^{*} \neq \varnothing$ for some $\gamma$-set $C^{*}$ of $H$. Then $|A|=1$. Let $w \in A$ and $p \in C \cap C^{*}$. Since $G=K_{n} \quad$ for $n>1$, there exists $u \in\left(V\left(K_{n}\right) \backslash\{w\}\right) \cap N_{G}(w)$ and $(u, v) \notin(A \times C)$ for all $v \in V(H)$. Thus, $(w, p)(u, v) \in E(G[H])$. Therefore $A \times C$ is a dominated superclique in $G[H]$.

Using Lemma 3, the proof of the next result will just be similar to that of Theorem 12 .

Theorem 13. Let $G=K_{n}$ for $n>1$ and $H$ a nontrivial connected graph with $\gamma(H)=1$. A subset $S$ of $V(G[H])$ is a restrained strong resolving dominating set of $G[H]$ if and only if $S=V(G[H]) \backslash(A \times C)$ and one of the following is satisfied:

(i) $A \subseteq V(G)$ and $C=\varnothing$

(ii) $A$ is nonempty nonsingleton subset of $V(G)$ and $C$ is a superclique in $H$ if $C \cap C^{*}=\varnothing$ for all $\gamma$-set $C^{*}$ in $H$. 
(iii) $A$ is a singleton subset of $V(G)$ and $C$ is a nonsingleton superclique in $H$ if $C \cap C^{*} \neq \varnothing$ for some $\gamma$-set $C^{*}$ of $H$.

Corollary 7. Let $G=K_{n}$ for $n>1$ and $H$ a non-trivial connected graph of order $m$. Then $\gamma_{r s R}(G[H])=m n-\omega_{D S}(G[H])$.

Proof: Let $S$ be a $\gamma_{r s R^{-}}$set of $G[H]$. Then $S$ is a restrained strong resolving dominating set of $G[H]$. By Theorem 12 or Theorem 13, $S=V(G[H]) \backslash(A \times C)$ where $A \times C$ is a dominated superclique in $G[H]$. Since $S$ is a $\gamma_{r s}$-set, $A \times C$ is a maximum dominated superclique of $G[H]$. Hence,

$$
\begin{aligned}
\gamma_{r s R}(G[H]) & =|S| \\
& =|V(G[H])|-|A \times C| \\
& =m n-\omega_{D S}(G[H]) .
\end{aligned}
$$

By Lemma 2 and Lemma 3, the dominated superclique $A \times C$ of $K_{n}[H]$ is maximum if $A=V(G)$ and $C$ is a maximum superclique in $H$. Thus, the next result follows.

Corollary 8. Let $G=K_{n}$ for $n>1$ and $H$ be a connected graph of order $m>1$. Then $\gamma_{r s R}(G[H])=n\left(m-\omega_{S}(H)\right)$.

\section{Acknowledgements}

This research is funded by the Department of Science and Technology - Accelerated Science and Technology Human Resource Development Program (DOST-ASTHRDP), Philippines.

\section{References}

[1] C. Berge. Theorie des graphes et ses applications. Metheun and Wiley, London and New York, 1962.

[2] Acal P.L. and Rara H.M. The Strong Connected Metric Dimension in the Join and Corona of Graphs. Advances and Applications in Discrete Mathematics, Vol 21 (2019), No. 1, 91-101. doi.org/10.17654/DM021010091.

[3] Acal P.L., Monsanto, G.B., and Rara H.M. On Strong Resolving Domination in the Join and Corona of Graphs European Journal of Pure and Applied Mathematics, Vol 13 (2020), No. 1, 170-179.

[4] Ibrahim T. A., and Omran A. A. Restrained Whole Domination in Graphs European Journal of Pure and Applied Mathematics, 1879 (2021) 032029

[5] Knor M., Tepeh H., and Skrekovski R. Domination Versus Independent Domination in Regular Graphs European Journal of Pure and Applied (2021). 
[6] Oellermann, O. R., and Peters-Fransen, J. (2007). The strong metric dimension of graphs and digraphs. Discrete Applied Mathematics, 155(3), 356-364.

[7] Domke, G.S., Hattingh, J.S, Hedetniemi, S.T., Laskar, R.C., and Markus, L.R. Restrained Domination in Graphs. Discrete Mathematics 203(1999) 61-69.

[8] P. Slater. Dominating and reference sets in a graph. Journal of Mathematics and Physical Science, 33(4):445-455, 1988.

[9] Harary, F. (1969). Graph theory. Michigan University Ann Arbor Department of Mathematics.

[10] Johnson, M. A. "Browsable structure-activity datasets." Advances in Molecular Similarity 2 (1998): 153-170.

[11] Khuller, Samir, Balaji Raghavachari, and Azriel Rosenfeld. Landmarks in graphs. Discrete Applied Mathematics 70.3 (1996): 217-229.

[12] Liu K. , Abu-Ghazaleh N. Virtual coordinate backtracking for void traversal in geographic routing. Lecture Notes in Comput. Sci., 4104 (2006), 46âAS59.

[13] Sebo, András and Tannier, Eric A. On metric generators of graphs. Mathematics of Operations Research, 29(2), 383-393.

[14] Bailey, Robert F., Cameron, Peter J. Base size, metric dimension and other invariants of groups and graphs. Base size, metric dimension and other invariants of groups and graphs, 43(2): 209-242,2011.

[15] Chartrand, G., Eroh, L., Johnson, M. A., and Oellermann, O. R. (2000). Resolvability in graphs and the metric dimension of a graph. Discrete Applied Mathematics, 105(13), 99-113.

[16] Chappell, G. G., Gimbel, J., and Hartman, C. (2008). Bounds on the metric and partition dimensions of a graph. Ars Combinatoria, 88, 349-366.

[17] Kuziak D., Yero I.G. and Rodriguez-Velasquez J.A. Closed Formuale for the Strong Metric Dimension of Lexicographic Product Graphs. Discussiones Mathematicae Graph Theory 36(2016) 1051-1064. doi:10.7151/dmgt.1911.

[18] Brigham, R.C., Chartrand G., Dutton, R.D., and Zhang P. Resolving Domination in Graphs. Mathematica Bohemica, Vol. 1 (2003), 25-36. 